\title{
SYSTEMS OF CONSERVATION LAWS WITH INVARIANT SUBMANIFOLDS
}

\author{
BY \\ BLAKE TEMPLE ${ }^{1}$
}

\begin{abstract}
Systems of conservation laws with coinciding shock and rarefaction curves arise in the study of oil reservoir simulation, multicomponent chromatography, as well as in the study of nonlinear motion in elastic strings. Here we characterize this phenomenon by deriving necessary and sufficient conditions on the geometry of a wave curve in order that the shock wave curve coincide with its associated rarefaction wave curve for a system of conservation laws. This coincidence is the one dimensional case of a submanifold of the state variables being invariant for the system of equations, and the necessary and sufficient conditions are derived for invariant submanifolds of arbitrary dimension. In the case of $2 \times 2$ systems we derive explicit formulas for the class of flux functions that give rise to the coupled nonlinear conservation laws for which the shock and rarefaction wave curves coincide.
\end{abstract}

Introduction. Systems of conservation laws which have coinciding shock and rarefaction curves arise in the study of oil reservoir simulation, nonlinear wave motion in elastic strings, as well as in multicomponent chromatography $[1,4,5,6,9,11,12]$. These systems have many interesting features. The Riemann problem for these equations can be explicitly solved in the large, and wave interactions have a simplified structure, even in the presence of a nonconvex flux function. For this reason, these systems represent some of the few examples for which the Cauchy problem has been solved for arbitrary data of bounded variation. Also, hyperbolic degeneracies appear in each of these systems. In the present paper we are concerned with characterizing the phenomenon of coinciding shock and rarefaction curves. This phenomenon turns out to be a special case of the general phenomenon of a submanifold of $\mathbf{R}^{n}$ being invariant for weak solutions of a given system of $n$ conservation laws. Invariant manifolds of higher dimension also appear in physical conservation equations in which the conserved quantities represent concentrations; i.e., when the concentrations $C_{i}$ of any of the species vanish, the system must reduce to a lower order system that expresses the conservation of the remaining species. Thus $C_{i} \equiv 0$ defines a manifold which is invariant in the sense that solutions that start on the manifold, remain there for all time. Moreover, such an invariant manifold of dimension $n-1$ is the boundary of an $n$-dimensional invariant region in the sense of Chueh, Conley and Smoller [14]. In the case of

Received by the editors December 20, 1982.

1980 Mathematics Subject Classification. Primary 35L65, 35L80; Secondary 76S05, 76T05.

${ }^{1}$ Research done in part while author was an NSF Postdoctoral Research Fellow visiting Rockefeller University (grant MCS8017157) and in part while author was a visiting member of the Courant Institute. 
concentration equations, this implies that $0 \leqslant C_{i} \leqslant 1$ is a bounded invariant region for general solutions. In $\S 3$ we give necessary and sufficient conditions for a submanifold to be invariant for weak solutions of a general system of conservation laws. The main result here is that an invariant manifold must be an affine linear space whenever no contact fields appear. For one-dimensional manifolds this implies that a shock curve can coincide with a rarefaction curve if and only if the rarefaction curve is either a straight line or the level curve of the corresponding wave speed. In $\$ 2$ we use these general results to explicitly write down the class of $2 \times 2$ conservation laws which have shock and rarefaction curves that coincide.

1. Preliminaries. A system of conservation laws in one space dimension is a set of partial differential equations of the form

$$
U_{t}+\widetilde{y}(U)_{x}=0 .
$$

Here $-\infty<x<\infty, t \geqslant 0$, and $U$ and $\widetilde{y}$ are vector-valued functions $U=$ $\left(u_{1}, \ldots, u_{n}\right)^{\mathrm{tr}} \equiv U(x, t), \widetilde{F}(U)=\left(f_{1}(U), \ldots, f_{n}(U)\right)^{\mathrm{tr}}$, where $U^{\mathrm{tr}}$ denotes the transpose of the vector $U$. We write $\widetilde{\mathcal{F}}(U)_{x}=d \widetilde{\mathcal{Y}} U_{x}$ where $d \widetilde{\mathscr{y}}$ denotes the matrix derivative of $\widetilde{y}$ with respect to $U$. The Cauchy problem is the natural problem to pose for system (1), and it is commonly known that discontinuities can form in the solutions of (1). For this reason we look for weak solutions $U(x, t)$; i.e., solutions that satisfy the following integral equation [7] for any smooth function $\psi(x, t)$ with compact support:

$$
\iint_{\substack{-\infty<x<+\infty \\ t \geqslant 0}} U \psi_{t}+\widetilde{\mathcal{F}}(U) \psi_{x}+\int_{-\infty}^{\infty} U(x, 0) \psi(x, 0) d x=0 .
$$

Two important systems of conservation laws arise in applications, and have been studied in $[1,4,5,6,9,11,12]$.

$$
\begin{gathered}
u_{t}+\{u \phi(u, v)\}_{x}=0, \\
v_{t}+\{v \phi(u, v)\}_{x}=0, \\
u_{t}+\left\{\frac{u}{1+u+v}\right\}_{x}=0, \\
v_{t}+\left\{\frac{\kappa v}{1+u+v}\right\}_{x}=0 .
\end{gathered}
$$

System (2) arises in problems of oil reservoir simulation, as well as in elasticity theory $[4,6,9,12]$. For example, in the reservoir simulation problem $[4,6], u$ is the saturation of water in the reservoir and $v$ is the concentration of a polymer in the water, so that $0 \leqslant u \leqslant 1,0 \leqslant v \leqslant 1$. The system is determined by specifying the function $\phi(u, v)$, but the structure of the solutions is determined by qualitative properties of $\phi$ which can be verified experimentally.

System (3) arises in the study of two component chromatography $[1,5,11]$. Here $u$ and $v$ are transformations of the concentrations of the two solutes, and $x$ and $t$ are transformations of the actual space and time variables (see Aris and Amundson [1, p.268]). The domains of the variables $u$ and $v$ can be taken to be $u \geqslant 0, v \geqslant 0$, and $\kappa \in(0,1)$ is determined by adsorption properties of the stationary phase. 
Systems (2) and (3) are remarkable because for both systems, the shock curves and rarefaction curves coincide. This leads us to study the phenomenon of coinciding shock and rarefaction curves in general. To say this precisely, assume first that system (1) is hyperbolic; i.e., that the eigenvalues (wave speeds) of $d \mathscr{F}$ are real but not necessarily distinct. Let $\lambda$ denote an eigenvalue, and $R$ a corresponding eigenvector of $d \mathscr{F}$. We call $(\lambda, \mathscr{R})$ a "characteristic family," or "characteristic field," for system (1) if $\lambda(U), \mathcal{R}(U)$ are defined and $C^{3}$ in some neighborhood $\Re$ of $U$-space. Let $\mathfrak{R} \subset \Re$ be the integral curve of $\Re$ through some point $U_{0} \in \mathfrak{N}$. $\Re$ is called the $\lambda$-rarefaction curve of $U_{0}$ in $\Re$. Rarefaction curves are the one-dimensional sets that smooth solutions to system (1) can take values on. For example, if the range of a smooth solution $U(x, t)$ of system (1) lies on a one-dimensional curve in $U$-space, then that curve must be a rarefaction curve. An analagous one-dimensional curve in $U$-space applies to the study of discontinuous solutions of (1). The Hugoniot locus of a point $U_{0}$ is defined to be the set of points $U$ such that

$$
\sigma[U]=[\mathscr{F}(U)]
$$

for some scalar $\sigma=\sigma\left(U, U_{0}\right),[U]=U-U_{0},[\mathscr{F}(U)]=F(U)-F\left(U_{0}\right)$. A state $U_{1}$ is in the Hugoniot locus of $U_{0}$ if and only if the discontinuous function

$$
U(x, t)= \begin{cases}U_{0} & \text { for } x<\sigma t \\ U_{1} & \text { for } x>\sigma t\end{cases}
$$

satisfies the weak form (1B) of system (1) [2,7]. Under very general conditions, there corresponds to each family $(\lambda, \Re)$ a one parameter subset of the Hugoniot locus of $U_{0}$ that has $C^{2}$ contact with the integral curve of $\Re$ at $U_{0}$ (cf. Lemma 3.1). This is called the $\lambda$-shock curve. (This term is often reserved for that portion of the curve that determines the physically acceptable solutions in (5).)

Definition 1. We say that the $\lambda$-shock curve coincides with the $\lambda$-rarefaction curve on $\Re$ if the Hugoniot locus of each point on $\Re$ contains $\Re$.

A curve $\mathfrak{N}$ on which the $\lambda$-shock and $\lambda$-rarefaction curves coincide is also a one-dimensional manifold that is invariant for weak solutions of the given system of conservation laws.

DEFINITION 2. We say that $\Re$ is a $k$-dimensional invariant manifold for system (1) (or equivalently that system (1) reduces to a $k \times k$ system of conservation laws on $\mathfrak{N}$ ) if, in a neighborhood $\Re$ of each point on $\mathfrak{N}$, there exists a coordinate system

$$
\phi: \mathbf{R}^{k} \rightarrow \mathbf{R}^{n}, \quad u \mapsto U,
$$

and a $k \times k$ strictly hyperbolic conservation law

$$
u_{t}+\overline{\mathscr{F}}(u)_{x}=0
$$

such that, for

$$
U(x, t)=\phi(u(x, t)) \subset \Re,
$$

$U(x, t)$ is a weak solution of (1) if and only if $u(x, t)$ is a weak solution of (2), (3). We assume only that system (7) is strictly hyperbolic (i.e. at each $u$ the matrix $d \overline{\mathscr{F}}(u)$ 
is assumed to have $k$ real and distinct eigenvalues) so that system (1) can have hyperbolic degeneracies.

Note that if or is a manifold on which system (1) reduces to a lower order system for all weak solutions, then any data $U_{0}(x) \equiv U(x, 0)$ given on $\Re$ at $t=0$, can be continued by (3) to a solution that remains on $\mathfrak{N}$ for all time, so long as system (2) has solutions. This explains the term invariant manifold.

In $\$ 3$ we state and prove Theorem 1 which gives necessary and sufficient conditions for a $k$-dimensional manifold $\mathbb{N}$ to be invariant for a given system of conservation laws. These general results imply the following theorem which characterizes the phenomenon of coinciding shock and rarefaction curves.

THEOREM 2. The following statements are equivalent regarding a $\lambda$-rarefaction curve $\because$ :

(i) The $\lambda$-shock and $\lambda$-rarefaction curves coincide on 912 .

(ii) Either $\lambda$ is constant on $)$, or on is a straight line in $U$-space.

(iii) System (1) reduces to a scalar conservation law on M. In this case weak solutions of (1) whose values lie on Mll are given by (8), where $u(x, t)$ is a weak solution of the scalar conservation law

$$
u_{t}+\lambda(\phi(u)) u_{x}=0
$$

and $\phi$ is a parameterization of $)$ with respect to some component of $U$.

We call $(\lambda, \leftrightarrow)$ a "contact" family if $\lambda$ is constant on each integral curve of $R$, and we call $(\lambda,(R)$ a "line" family if each integral curve of $(h$ is a straight line in $U$-space. In the next section we derive the class of $2 \times 2$ equations that have either a contact or a line family. (In this case we let $U \equiv(u, v)$ and for convenience we assume that a contact family satisfies $\nabla \lambda=(\partial \lambda / \partial u, \partial \lambda / \partial v) \neq 0$ in $: r$, and that a line family satisfies $\nabla q \neq 0$ in $\cdot \pi$, where $q(u, v)$ is the slope $d v / d u$ of the integral curve of $R$ through $(u, v)$. Weaker assumptions can be made.) All of the characteristic families in systems (2) and (3) are then seen to be either line or contact families. In this way the phenomenon of coinciding shock and rarefaction curves is observed from the explicit form of the equations.

2. Coinciding shock and rarefaction curves for $2 \times 2$ systems. Consider an arbitrary system of $2 \times 2$ conservation laws

$$
u_{t}+f(u, v)_{x}=0, \quad v_{t}+g(u, v)_{x}=0,
$$

where we take $U \equiv(u, v), \widetilde{y}=(f, g)$. We now locate the class of such $2 \times 2$ equations that have either a contact or a line field in a region $\mathcal{X}$ of $U$-space. These are generically the only fields that have coinciding shock and rarefaction curves, as indicated by Theorem 2 . The proof of Theorem 2 is postponed until the next section.

We let $q=q(u, v)$ denote a Riemann invariant for a contact or a line field in $\mathcal{R}$. A Riemann invariant for a family $(\lambda, \mathscr{R})$ is a function which is constant on the integral curves of $R$. We assume that $\nabla q \neq 0$, and because it suffices to prove our results locally, we always assume that $q=$ const determines a unique integral curve of $R$ in $\mathcal{N}$, which has a finite slope $d v / d u$. 
First assume that $q(u, v)$ is the wave speed of a contact field for a system of $2 \times 2$ equations, $\nabla q \neq 0$. The function $q$ is thus a Riemann invariant for the contact field

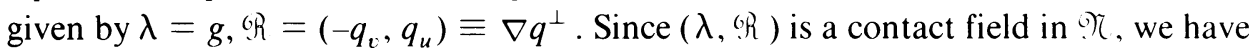
by Theorem 2 that the $\lambda$-shock curves coincide with the $\lambda$-rarefaction curves in $\gamma$, so the curve $q=$ const must be contained within the Hugoniot locus of every point on that curve. Thus by (4), if $[q] \equiv q(U)-q\left(U_{0}\right)=0$, then also

$$
\sigma[u]=[f], \quad \sigma[v]=[g],
$$

and in the contact case, $\sigma \equiv \sigma\left(U, U_{0}\right)=q\left(U_{0}\right)$ (cf. [7]). Conversely, if (2.2) holds in $\Re$ when $[q]=0$ for some smooth function $q(u, v), \nabla q \neq 0$, then $\left(\sigma\left(U_{0}, U_{0}\right)\right.$, $\left.\nabla q^{\perp}\left(U_{0}\right)\right)$ must be a characteristic field for $d \mathscr{F}$. To see this, note that (2.2) implies that

$$
\sigma\left(U_{0}, U_{0}\right)=\lim _{U \rightarrow U_{0}} \frac{[f]}{[u]}=f_{u}+f_{v} \frac{d v}{d u}=\lim _{U \rightarrow U_{0}} \frac{[g]}{[v]}=g_{v}+g_{u} \frac{d u}{d v},
$$

where the vector $(1, d v / d u)$ is parallel to $\nabla q\left(U_{0}\right)$.

Therefore, we can verify that

$$
d \widetilde{F} \cdot\left(1, \frac{d v}{d u}\right)^{\mathrm{tr}}=\sigma\left(U_{0}, U_{0}\right)\left(1, \frac{d v}{d u}\right) .
$$

Thus the statement that (2.2) holds with $\sigma=q$ when $[q]=0$ in $\Re$, is equivalent to the statement that $(\lambda, \mathscr{R})=\left(q, \nabla q^{\perp}\right)$ is a contact field in $\Re$. But (2.2) holds when $[q]=0$ if and only if

$$
[f-u g]=0, \quad[g-v q]=0,
$$

when $[q]=0$; and (2.4) holds if and only if $f=u q+F(q)$ and $g=v q+G(q)$ for some smooth functions $F$ and $G$. We have the following

TheOREM 3. A system of $2 \times 2$ conservation laws (2.1) has a contact field in a domain $\mathfrak{K}$ of $u v$-space if and only if $f$ and $g$ satisfy

$$
f(u, v)=u q+F(q), \quad g(u, v)=v q+G(q),
$$

in $\mathcal{\gamma}$, for some smooth functions $q, F$ and $G, \nabla q \neq 0$. In this case $q(u, v)=\lambda$ where $\lambda$ is the wave speed of the contact family.

Next assume that $(\lambda, \Re)$ is a line family for a system of $2 \times 2$ equations defined in a region $\mathcal{R}$ of $U$-space. Let $q(u, v)$ be the slope $d v / d u$ of the integral curve of $R$ through the point $(u, v) \in \mathfrak{N}, \nabla q \neq 0$. The function $q$ is a Riemann invariant of $R$. Moreover, $q$ is a smooth nonconstant solution to Burger's equation

$$
q_{u}+q q_{v}=0
$$

since $\nabla q$ is orthogonal to the vector $(1, q)$ at every point in $\Re$. Since $(\lambda, \Re)$ is a line field, Theorem 2 again implies that a curve defined by $q=$ const contains the Hugoniot locus of each point on that curve. Thus, when $[q]=0$,

$$
\sigma[u]=[f], \quad \sigma[v]=[g] .
$$

Dividing we obtain that, when $[q]=0$,

$$
q=[v] /[u]=[g] /[f],
$$


so that

$$
[f q-g]=0 .
$$

By (2.3) this is equivalent to the statement that $(\lambda$, o $R)$ is a line field in $\mathscr{\gamma}$. But (2.8) holds if and only if $g=f q+H(q)$ for some smooth function $H$. We have proven the following.

THEOREM 4. A system of $2 \times 2$ conservation laws has a line family in $\mathcal{X}$ if and only if f and $g$ satisfy

$$
g=f q+H(q)
$$

in $\cdot)$, for some smooth function $H$, where $q(u, v)$ is a smooth solution to Burger's equation (2.6) with $\nabla q \neq 0$. In this case $q$ is a Riemann invariant for the line family.

Theorem 3 applies to system (2) with $F=G \equiv 0$, and thus $\left(\phi, \nabla \phi^{\perp}\right)$ must be a contact family for system (2). Since system (2) also satisfies

$$
g(u, v)=v \phi(u, v)=(v / u) f(u, v),
$$

and $v / u$ is a smooth solution to Burger's equation in $u>0, v>0$, we have by Theorem 4 that $q(u, v)=v / u$ is the Riemann invariant of a line family for system (2). Moreover, we can use Theorems 3 and 4 to locate the class of $2 \times 2$ equations that have both a line and a contact field; i.e., we say that system (1) has both a contact and a line field in $\mathcal{N}$ if system (1) has two Riemann invariants $q$ and $p$ that satisfy Theorems 3 and 4 respectively, such that $u$ and $v$ are smooth functions of $(p, q)$ off a closed set of measure zero in $\mathcal{X}$. By Theorems 3 and 4 ,

$$
v q+G(q)=u q p+F(q) p+H(p) .
$$

Formally differentiating (2.10) with respect to $u$ and holding $p$ fixed yields

$$
v+G^{\prime}(q)=u p+F^{\prime}(q) p
$$

since $(\partial / \partial u) v(u, p)=p$ because $p$ is a smooth solution of (2.6). Differentiating (2.10) with respect to $q$ and holding $p$ fixed gives

$$
\frac{\partial}{\partial q} v(p, q)+v+G^{\prime}(q)=u p+\frac{\partial}{\partial q} u(p, q) q p+F^{\prime}(q) p .
$$

Therefore, substituting (2.11) into (2.12) we obtain

$$
\frac{\partial}{\partial q} v(p, q)=p \frac{\partial}{\partial q} u(p, q)
$$

Now differentiate (2.11) with respect to $q$ holding $p$ fixed and obtain

$$
\frac{\partial}{\partial q} v(p, q)+G^{\prime \prime}(q)=p \frac{\partial}{\partial q} u(p, q)+F^{\prime \prime}(q) p
$$

which by (2.13) is

$$
G^{\prime \prime}(q)=F^{\prime \prime}(q) p .
$$

Finally, differentiating (2.15) with respect to $p$ and holding $q$ fixed, we conclude

$$
F^{\prime \prime}(q)=G^{\prime \prime}(q)=0 \text {, }
$$

or

$$
F(q)=a q+c, \quad G(q)=b q+d
$$


for some constants $a, b, c, d$. The assumptions made in (2.11)-(2.17) are that $v$ is a differentiable function of $(u, p),(\partial / \partial u) q(u, p) \neq 0$, and $q \neq 0$. But by (2.6) these must hold off a closed set of measure zero in $\Re$, so (2.17) must hold everywhere in $\mathfrak{X}$. Moreover, since the addition of a constant to the flux functions $f$ and $g$ in (2.1) does not affect the solutions, we can take $c=d=0$. Substituting (2.17) into (2.11) then gives $p=p(u, v)=(v+b) /(u+q)$, and the constraint in (2.10) yields

$$
H(p)=-b p \text {. }
$$

We have thus proven the following.

COROLlaRy 1. System (2.1) has both a line and a contact field if and only if

$$
f=(u+a) q, \quad g=(v+b) q .
$$

for some smooth function $q=q(u, v)$, and some constants $a$ and $b$. In this case $q$ is the wave speed of the contact family, and

$$
p=(v+b) /(u+a)
$$

is the Riemann invariant of the line field satisfying (2.6),

Now consider an arbitrary $2 \times 2$ system that has two distinct line families. By Theorem 3, there exist two distinct solutions $p$ and $q$ of Burger's equation such that (2.9) holds; i.e., such that

$$
\begin{aligned}
& g=f p+H_{1}(p), \\
& g=f q+H_{2}(q),
\end{aligned}
$$

for some smooth functions $H_{1}$ and $H_{2}$. Equating (2.10) and (2.11) gives

$$
f=\frac{H_{1}(p)-H_{2}(q)}{q-p} .
$$

This proves the following.

COROLlary 2. System (2.1) has two line families if and only if

$$
f=\frac{H_{1}(p)-H_{2}(q)}{q-p}, \quad g=\frac{g H_{1}(p)-p H_{2}(q)}{q-p},
$$

where $p$ and $q$ are smooth solutions of (2.6).

For system (3) one can verify that

$$
\frac{u}{1+u+v}=\frac{H(p)-H(q)}{q-p}, \quad \frac{K v}{1+u+v}=\frac{q H(p)-p H(q)}{q-p},
$$

where

$$
H(z)=\frac{z-\kappa z}{z+1},
$$

and $p, q$ are the two solutions of Burger's equation which satisfy

$$
u z^{2}+\{\kappa(u+1)-(v+1)\} z-\kappa v=0
$$

in $z$, and are smooth in $u>0, v>0$. This verifies that system (3) has a pair of line families with integral curves given by $p=$ const and $q=$ const. 
Finally, note that a system of $2 \times 2$ equations generically has two characteristic families. The functions $f$ and $g$ given in (2.5) and (2.9) involve explicitly a Riemann invariant of one family. The following result, which is easily verified, determines both characteristic families given a Riemann invariant of one family. This general result is simple and explains many of the calculations in $[4,12]$.

Theorem 5. Let $q=q(u, v)$ be a Riemann invariant for a system of $2 \times 2$ conservation laws $(2.1)$, and let $\bar{f}(u, q)=f(u, v)$ and $\bar{g}(v, q)=g(u, v)$ be smooth. Then $\left(\lambda_{i}, \leftrightarrow_{i}\right), i=1,2$, are characteristic fields for the system, where

$$
\begin{gathered}
\lambda_{1}=\bar{f}_{u}, \quad \Re_{1}=\nabla q^{\perp}, \\
\lambda_{2}=\bar{f}_{u}+\bar{f}_{q} q_{u}+\bar{g}_{q} q_{v}, \quad \Re_{2}=\left(\bar{f}_{q}, \bar{g}_{q}\right) .
\end{gathered}
$$

3. In this section we prove Theorems 1 and 2 which give necessary and sufficient conditions on the geometry of $: \pi$ for $: \pi$ to be an invariant manifold for system (1). Note that Definition 2 is local and locally $: \pi$ can be parameterized by $k$ coordinates of $U$, say $u \equiv\left(u_{1}, \ldots, u_{k}\right)^{\text {tr: }}$ :

$$
U \equiv\left(u_{1}, \ldots, u_{n}\right)^{\operatorname{tr}}=\left(u_{1}, \ldots, u_{k}, \phi\left(u_{1}, \ldots, u_{k}, \chi\left(u_{1}, \ldots, u_{k}\right)\right)\right)^{\operatorname{tr}}=\phi(u) .
$$

We assume that the transformation in (6) is given by (3.1), and that the flux function in (7) is given by

$$
\overline{\widetilde{F}}(u)=\text { first } k \text { rows of }\{\widetilde{F} \cdot \phi(u)\} \text {. }
$$

This is reasonable since solutions of (1) must satisfy this $k \times k$ system in the first $k$ equations. That it actually represents no loss of generality can be shown by carrying out the following analysis with a general transformation $\phi$.

We say that $M$ is invariant for smooth solutions of systems (1) if Definition 2 holds for all smooth solutions $U(x, t)=\phi(u(x, t))$ of system (1). The first lemma gives necessary and sufficient conditions for $\mathfrak{M}$ to be invariant for smooth solutions of system (1).

LEMMA 3.0. Ar is a k-dimensional manifold which is invariant for smooth solutions of system (1) if and only if $\mathcal{M}$ is an integral manifold of $k$ eigenvectors for distinct eigenvalues of $d \widetilde{T}$.

Proof. First assume that $) \pi$ is an integral manifold of $k$ linearly independent eigenvectors $R_{1}, \ldots, R_{k}$ of $d \mathscr{F}$. We show that for $U(x, t)=\phi(u(x, t)), U$ is a smooth solution of (1) if and only if $u$ is a smooth solution of (7), where $\overline{\mathcal{F}}$ is defined in (3.2).

Let $A$ be the $n \times k$ matrix given by

$$
d \phi \equiv A \equiv\left[\begin{array}{c}
I_{k} \\
B
\end{array}\right],
$$

where $I_{k}$ is the $k \times k$ identity matrix. Let $A_{1}, \ldots, A_{k}$ be the columns of $A$. Each $A_{i}$ lies in the span of the $k$ eigenvectors of the matrix $d \mathscr{F}$ at each value of $u$. Define the $n \times k$ matrix $R$ by

$$
R \equiv\left[\Re_{1}, \ldots, \Re_{k}\right]
$$


Since each $A_{i}$ is in the span of ${ }_{R} R_{1}, \ldots, \Re_{k}$, there exists a $k \times k$ nonsingular matrix $D$ such that

$$
A D=R .
$$

Finally, define the $k \times k$ matrix $\lambda$ by

$$
\lambda=\left[\begin{array}{ccc}
\lambda_{1} & & 0 \\
& \ddots & \\
0 & & \lambda_{k}
\end{array}\right],
$$

where $\lambda_{1}, \ldots, \lambda_{k}$ are the $k$ eigenvalues of ${ }^{2} R_{1}, \ldots, R_{k}$, respectively. Then we can write

$$
d \widetilde{\mathscr{Y}} A=d \widetilde{\mathscr{Y}} R D^{-1}=R \lambda D^{-1}=A D \lambda D^{-1} .
$$

We show first that the matrix $D$ satisfies

$$
d \bar{\sigma}=D \lambda D^{-1},
$$

so that the columns of $D$ are eigenvectors of the matrix $d \overline{\mathscr{F}}$; i.e., $d \overline{\bar{y}}$ is the $k \times k$ matrix given by the first $k$ rows of the $n \times k$ matrix

$$
d(\mathscr{F}(\phi(u)))=d \mathscr{F} d \phi=d \mathscr{F} A \text {. }
$$

But by (3.7) we have

$$
d^{\mathscr{F}} A=A D \lambda D^{-1} .
$$

Since $A$ is the identity in the first $k$ rows,

$$
d \overline{\widetilde{\mathscr{F}}}=1 \text { st } k \text { rows of }\{d \widetilde{\mathscr{F}} A\}=1 \text { st } k \text { rows of }\left\{A D \lambda D^{-1}\right\}=D \lambda D^{-1} \text {, }
$$
verifying (3.8).

Now $U(x, t)=\phi(u(x, t))$ satisfies system (1) if and only if $u(x, t)$ satisfies

$$
A u_{t}+d \mathscr{F} A u_{x}=0,
$$

or by (3.7), (3.8), if and only if

$$
A\left(u_{t}+d \overline{\mathscr{F}} u_{x}\right)=0
$$

which holds if and only if

$$
u_{i}+\overline{\mathscr{F}}(u)_{x}=0
$$

since the rank of $A$ is $k$.

Conversely, now assume that for $U=\phi(u), U(x, t)$ is a smooth solution of (1) if and only if $u(x, t)$ is a smooth solution of the $k \times k$ strictly hyperbolic system

$$
u_{t}+\overline{\mathscr{F}}(u)_{x}=0
$$

where $\phi$ is a smooth map

$$
\phi: \mathbf{R}^{k} \rightarrow \mathbf{R}^{n} .
$$

We show that $\mathscr{R}=\{U: U=\phi(u)\}$ is an integral manifold of $k$ eigenvectors of $d \mathscr{F}$. Again let $A$ denote the $n \times k$ matrix

$$
A=d \phi
$$


and let $l_{1}, \ldots, l_{k}$ be the $k$ independent left eigenvectors of $d \overline{\mathscr{F}}$. Define the $k \times k$ matrix $L$ by

$$
L=\left[\begin{array}{c}
l_{1} \\
\vdots \\
l_{k}
\end{array}\right] .
$$

Since $A, L$ have rank $k$, there exists an $n \times k$ matrix $E$ and a $k \times n$ matrix $E^{-1}$ such that

$$
A=E L
$$

and

$$
E^{-1} A=L .
$$

Since smooth solutions $U(x, t)=\phi(u(x, t))$ satisfy system (1) if and only if $u(x, t)$ satisfies system (2), we must have that smooth functions $u(x, t)$ satisfy

$$
A u_{t}+d \mathscr{F} A u_{x}=0
$$

if and only if they also satisfy

$$
A u_{t}+A d \overline{\mathscr{F}} u_{x}=0
$$

This implies that

$$
d \widetilde{\mathscr{F}} A=A d \overline{\mathscr{F}} .
$$

From (20) and (21) we obtain

$$
A d \overline{\mathscr{F}}=E L d \overline{\mathscr{F}}=E \lambda L=E \lambda E^{-1} A .
$$

Here $\lambda$ is the matrix given in (3.8) where the $\lambda_{i}$ are the eigenvalues associated with eigenvectors $l_{i}$. Substituting into (3.23) we obtain

$$
\left(d \mathscr{F}-E \lambda E^{-1}\right) A=0 .
$$

Multiplying (3.25) on the left by $E^{-1}$ we have

$$
\left(E^{-1} d \mathscr{F}-\lambda E^{-1}\right) A=0 .
$$

But $\left(E^{-1} d \mathscr{F}-\lambda E^{-1}\right)$ is a $(k \times n)$ matrix, and (3.26) gives $k$ independent linear combinations of the columns of this matrix that are zero. Thus

$$
\operatorname{rank}\left(E^{-1} d \mathscr{F}-\lambda E^{-1}\right)=0,
$$

so we must have

$$
E^{-1} d \mathscr{F}-\lambda E^{-1}=0 .
$$

Multiplying (3.28) on the left and right by the $n \times k$ matrix $D$ yields

$$
d \mathscr{F} E=E \lambda,
$$

so the $k$ columns of $E$ are $k$ independent eigenvectors of $d \mathscr{F}$. By (3.19) and (3.20), the columns of $A$ lie in the span of the columns of $E$. Therefore, since $A=d \phi, \Re$, the image of $\phi$, must be an integral manifold for $k$ independent eigenvectors of $d F$. This completes the proof of Lemma 3.0. 
We are now interested in obtaining necessary and sufficient conditions on the geometry of $\mathfrak{N}$ in order that $\mathfrak{N}$ be an invariant submanifold for weak solutions of system (1). Such an $\mathfrak{N}$ must first be invariant for smooth solutions of (1), so without loss of generality we assume that $\mathfrak{N}$ is the integral manifold for $k$ independent eigenvectors $\mathscr{R}_{1}, \ldots, \Re_{k}$ of $d \mathscr{F}$. In this case, from (3.8) we have that $\Re_{i}=d \phi \overline{\mathscr{G}}_{i}$ where $\bar{G}_{i}$ is the eigenvector of $d \overline{\overline{\mathscr{F}}}$ associated with the eigenvalue $\lambda_{i}$. Thus, letting $\Re_{i}\left(u_{L}\right)$ denote the integral curve of $\bar{\Re}_{i}$ through $u_{L} \in \mathbf{R}^{k}$, we have that $\phi \cdot G_{i}\left(u_{L}\right)$ is the integral curve of $\Re_{i}$ through $U_{I}=\phi\left(u_{L}\right)$. We now study the Hugoniot loci for the $k \times k$ strictly hyperbolic system (7). Lemma 3.1 gives a local characterization of this loci (cf. [7, 13]).

LEMMA 3.1. In a neighborhood of each point in $\mathbf{R}^{k}$, the Hugoniot locus of each state $u_{L}$ consists of $k$ smooth curves $S_{i}\left(u_{L}\right), i=1, \ldots, k$, such that $S_{i}\left(u_{L}\right)$ makes $C^{2}$ contact with the integral curve $\Re_{i}\left(u_{l}\right)$ at $u_{l}$.

We call $S_{i}\left(u_{L}\right)$ the $i$-shock curve and we call $\Re_{i}\left(u_{L}\right)$ the $i$-rarefaction curve associated with the point $u_{L}$. For such curves we let $S_{L}^{i}(\xi)$ denote some regular parameterization of $S_{i}\left(u_{L}\right)$ with $S_{L}^{i}(0)=u_{L}$, and we let $\lambda_{L}^{i}(\xi)$ be the value of $\lambda_{i}$ at $S_{I}^{i}(\xi)$. If $S_{i}\left(u_{L}\right)=\Re_{i}\left(u_{L}\right)$, then we say that the $i$-shock and rarefaction curves coincide, and if further $d \lambda_{L}^{i}(\xi) / d \xi \equiv 0$, then we call $S_{i}\left(U_{L}\right)$ a contact curve. It is easily verified that if $d \lambda_{L}^{i}(\xi) / d \xi=0$ for $\xi \in(a, b)$ where $(a, b)$ is any open interval in the domain of $\xi$, then $S_{L}^{i}(\xi)$ simply parameterizes that rarefaction curve $\Re_{i}(a)$ between $S_{L}^{i}(a)$ and $S_{L}^{i}(b)$.

Now let $S\left(U_{L}\right)$ denote any smooth curve through $U_{L} \in \mathbf{R}^{n}$ such that $S\left(U_{L}\right)$ is a subset of the Hugoniot locus of $U_{L}$ for system (1), and let $S_{L}(\xi)$ denote a regular parameterization of this curve, $S_{L}(0)=U_{L}$.

LEMMA 3.2. If $S\left(U_{L}\right) \subset \mathfrak{N}$ in a neighborhood $\Re$ of $U_{L}$, then in $\Re$, both

$$
S\left(U_{L}\right)=\phi \circ S_{i}\left(u_{L}\right)
$$

for some $i \in\{1, \ldots, k\}$, and

$$
\left\{\frac{d}{d \xi} \lambda_{L}^{i}(\xi)\right\} \cdot\left\{\left(S_{L}(\xi)-U_{L}\right) \circ N\right\} \equiv 0
$$

for every $N \in\left\{T_{U} \Re\right\}^{\perp}$, where $\left\{T_{U} \Re\right\}^{\perp}$ denotes the orthogonal complement of the tangent space of T at $U=S_{L}(\xi)$.

Statement (3.31) states simply that whenever $\lambda_{i}$ is not constant on $S\left(U_{L}\right)$, the vector $\left(S_{L}(\xi)-U_{L}\right)$ must lie in the tangent space of $\mathscr{T}$ at $S_{L}(\xi)$.

Proof. Assume that $S\left(U_{L}\right) \subset \Re$. Then each point $U_{R} \in S\left(U_{L}\right)$ is in the Hugoniot locus of $U_{L}$ for the system (1), and $U_{R}$ is also in the Hugoniot locus of $U_{L}$ for the system (7) which is just the first $k$ equations in system (1). Therefore, by Lemma 3.1, $S\left(U_{L}\right)=\phi \circ S_{i}\left(u_{L}\right)$ in some neighborhood of $U_{L}$, some $i=1, \ldots, k$. Without loss of generality, assume that values of $U$ lie in a neighborhood $\Re$ of $U$-space where the shock curve $S_{i}\left(u_{L}\right)$ is regularly parameterized by $u_{1} \equiv \xi$, and write

$$
S_{L}^{i}(\xi)=(\xi, H(\xi), \chi(\xi, H(\xi)))=\phi(\xi, H(\xi)),
$$


where $H=\left(H_{2}, \ldots, H_{k}\right)$ and $u_{i}=H_{i}(\xi), i=2, \ldots, k$, defines the curve $S_{i}\left(u_{l}\right)$ in the first $k$ variables. Let

$$
f(\xi) \equiv f_{1}(\phi(\xi, H(\xi))), \quad \phi(\xi) \equiv \phi(\xi, H(\xi)) .
$$

Then for $U$ on $S\left(U_{l}\right)$ we must have

$$
\boldsymbol{\sigma}[U]=[\overline{\mathrm{F}}], \quad \boldsymbol{\sigma}=[f] /[\xi],
$$

where $[U]=U-U_{L},[\widetilde{\mathscr{Y}}]=\widetilde{\mathscr{Y}}(U)-\widetilde{\mathscr{Y}}\left(U_{l}\right)$, etc. Thus

$$
[\widetilde{\sigma}]=\frac{[f]}{[\xi]}[\phi] \text {. }
$$

Differentiating both sides of (3.35) with respect to $\xi$ gives

$$
f^{\prime} \frac{[\phi]}{[\xi]}+\frac{[f]}{[\xi]} \phi^{\prime}-\frac{[f]}{[\xi]} \frac{[\phi]}{[\xi]}=d \bar{\varphi} \cdot \phi^{\prime},
$$

where $f^{\prime} \equiv f^{\prime}(\xi), \phi^{\prime} \equiv \phi^{\prime}(\xi)$, and $d \widetilde{q}$ is evaluated at $\phi(\xi)$. Now let $\left\{N_{1}, \ldots, N_{n-k}\right\}$ be an orthonormal basis for the orthogonal complement of the tangent space of $: \pi$ at $U=\phi(\xi)$. Since $\phi^{\prime}$ by assumption lies in $T_{U}$, Ml we have $N_{j} \cdot \phi^{\prime}=0$ for $j=1, \ldots$, $n-k$. Moreover

$$
N_{j} \cdot\left(d \widetilde{{ }^{\top}} \phi^{\prime}\right)=N_{j} \cdot\left(\lambda_{1} C_{1} \mathscr{Q R}_{1}+\cdots+\lambda_{k} C_{k} \cdot Q_{k}\right)=0,
$$

where $\phi^{\prime}(\xi)=C_{1}\left(\Re_{1}(U)+\cdots+C_{k} \Re_{k}(U)\right.$ gives $\phi^{\prime}$ as a linear combination of the eigenvectors ${ }^{R}{ }_{i}(U)$. Thus from (3.36) we obtain

$$
\left\{[f]-f^{\prime}[\xi]\right\}\left\{N_{j} \cdot[\phi]\right\} \equiv 0
$$

for $j=1, \ldots, n-k$. If $[f]-f^{\prime}[\xi]=0$, then

$$
[f] /[\xi]=f^{\prime}
$$

and so from (3.36) we obtain

$$
f^{\prime} \phi^{\prime}=d \widetilde{\mathscr{\gamma}} \phi^{\prime}
$$

in which case $\phi^{\prime}$ must be an eigenvector of $d F$ with eigenvalue $f^{\prime}$. But $S_{I}(\xi)=\phi$. $S_{L}^{i}(\xi)$ has $C^{2}$ contact with $\Re_{i}=\phi \cdot \bar{R}_{i}$ at $\xi=0$. Therefore, in the case of (3.40), $\phi^{\prime}=\Re_{i}$ and $f^{\prime}=\lambda_{i}$ near $U_{L}$. Statement (3.39) then reads

$$
\lambda_{L}^{i}(\xi)=[f] /[\xi],
$$

which, when differentiated, yields

$$
\frac{d}{d \xi} \lambda_{L}^{i}(\xi)=\frac{f^{\prime}-[f] /[\xi]}{[\xi]}=0 .
$$

Substituting back into (3.38) yields

$$
\left\{\frac{d}{d \xi} \lambda_{L}^{i}(\xi)\right\} \cdot\left\{N_{j} \cdot\left(S_{L}(\xi)-U_{L}\right)\right\} \equiv 0
$$

which proves Lemma 3.2. The first application of Lemma 3.2 characterizes invariant manifolds in the case when $\mathscr{R}$ is the integral manifold of $k$ eigenvectors $\mathfrak{R}_{1}, \ldots, \Re_{k}$ none of which is a contact field. 
THEOREM 1. Assume that $\nabla \lambda_{i} \cdot R_{i}=0$ on at most a set of measure zero on each integral curve of $\Omega_{i}, i=1, \ldots, k$. Then the following statements are equivalent regarding an integral manifold $\Re$ of the eigenvectors $\Re_{1}, \ldots, \Re_{k}$ :

(i) For every $U_{L}=\phi\left(u_{L}\right) \in \mathfrak{M}$, the curves $\phi \cdot S_{i}\left(u_{L}\right)$ lie in the Hugoniot locus of $U_{L}=\phi\left(u_{L}\right)$ for system (1).

(ii) $\mathfrak{N}$ is a $k$-dimensional affine linear space in $U$-space.

(iii) $\Re$ reduces to the $k \times k$ system (7) on $\Re(i . e ., ~ \Re($ is a $k$-dimensional invariant manifold).

Proof. First we show that (i) implies (ii). So assume (i) and suppose that $\Re$ is not an affine linear space in $U$-space. Note first that by Lemma 3.1, the $k$ vectors $\left\{d S_{L}^{i}(\xi) / d \xi\right\}$ span $\mathbf{R}^{k}$ at $\xi=0$ for every $u_{L} \in \mathbf{R}^{k}$. Thus the vectors $\left\{d \phi \cdot d S_{L}^{i}(0) / d \xi\right\}$ span the tangent space of $\Re$ at each $U_{L}=\phi\left(u_{L}\right)$. Thus if $\Re$ is not an affine linear space, then there must exist a point $U_{L}$ and an $i \in\{1, \ldots, k\}$ such that $S(\xi)=\phi$. $S_{L}^{i}(\xi)$ satisfies

$$
S^{\prime \prime}(0) \cdot N \neq 0
$$

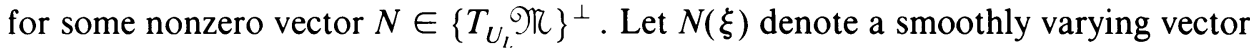
on $\xi$ such that $N(0)=N$ and $N(\xi) \in\left\{T_{\gamma(\xi)} \Re\right\}^{\perp}$. Then by continuity we have that

$$
\frac{S^{\prime}(\xi)-(S(\xi)-S(0)) / \xi}{\xi} \cdot N(\xi) \neq 0
$$

in a neighborhood of $\xi=0$. Since $S^{\prime}(\xi) \cdot N(\xi)=0$, we conclude that

$$
[S(\xi)-S(0)] \cdot N(\xi) \neq 0
$$

in some deleted neighborhood of $\xi=0$. This contradicts Lemma 3.2 since $d \lambda_{L}^{i}(\xi) / d \xi=0$ only on a set of measure zero. Therefore we have that (i) implies (ii).

Now assume (ii). Then $U=A u$ defines the map $\phi$, where $A$ is a constant $k \times n$ matrix of the form (3.3), where $B$ is a constant $k \times(n-k)$ matrix. Thus by (3.13), system (1) is equivalent to the two systems

$$
\begin{gathered}
u_{t}+d \overline{\mathscr{F}}(u) u_{x}=0, \\
B u_{t}+B d \overline{\mathscr{F}}(u) u_{x}=0,
\end{gathered}
$$

where $\overline{\mathscr{F}}(u)$ is given in (3.2). Since $B$ is constant, weak solutions of (3.47) satisfy (3.48) weakly, which verifies that $U$ is a weak solution of (1) if and only if $u$ is a weak solution of (7). This is (ii).

Finally, (i) immediately follows from (iii) since the curves $S_{i}\left(u_{L}\right)$ determine weak solutions of system (7) of the form (5). This completes the proof of Theorem 1.

Proof of Theorem 2. Let $\phi:\left[\xi_{1}, \xi_{2}\right] \rightarrow \Re$ be a local parametrization of the integral curve $\mathscr{R}$ of $\Re_{i}$ with respect to some coordinate $u_{i} \equiv u \equiv \xi$. Let $\lambda(\xi)$ be the corresponding eigenvalue. We show first that (i) implies (ii). So assume that (i) holds. By Lemma 3.2 we have that

$$
\lambda^{\prime}(\xi) \cdot\left\{\left(\phi(\xi)-\phi\left(\xi_{0}\right)\right) \cdot N\right\}=0,
$$

for every $\xi, \xi_{0} \in\left[\xi_{1}, \xi_{2}\right]$ and every vector $N$ satisfying

$$
N \cdot \phi^{\prime}(\xi)=0 .
$$


Note that if the term in brackets in (3.49) vanishes on an open set in $\left[\xi_{1}, \xi_{2}\right]$, then $\phi^{\prime \prime} \equiv 0$ on that set. Suppose now that it is not the case that either $\phi_{N}^{\prime \prime} \equiv 0$ or $\lambda^{\prime} \equiv 0$ in $\left[\xi_{1}, \xi_{2}\right]$, where $\phi_{N}^{\prime \prime}$ denotes the component of $\phi^{\prime \prime}$ normal to the curve $\mathfrak{N}$. Then by (3.49) there must exist a point $\xi$ and an open interval $\Phi_{0}$ in $\left[\xi_{1}, \xi_{2}\right]$ such that

$$
\begin{array}{rlrl}
\lambda^{\prime}(\xi) \neq 0, & & \lambda^{\prime}\left(\xi_{0}\right)=0, \\
\phi^{\prime \prime}(\xi)=0, & \phi_{N}^{\prime \prime}\left(\xi_{0}\right) \neq 0
\end{array}
$$

for all $\xi_{0} \in g_{0}$. Statement (3.52) implies that $\left\{\left(\phi(\xi)-\phi\left(\xi_{0}\right)\right) \cdot N\right\} \neq 0$ for some $\xi_{0} \in \mathscr{g}_{0}, N \in \operatorname{Span}\left\{\phi^{\prime}(\xi)\right\}^{\perp}$. This contradicts (3.44) since we also have $\lambda^{\prime}(\xi) \neq 0$. Thus (i) implies (ii).

Now assume (ii). If $\mathfrak{T}$ is a straight line then we are in the case of Theorem 2. So assume $\lambda$ is constant on $\mathfrak{R}$. Since $u \equiv u_{i}$,

$$
\frac{d}{d u} \mathcal{F} \cdot \phi(u)=d \mathscr{F} \cdot \phi^{\prime}(u)=\lambda \phi^{\prime}(u),
$$

and since $\lambda$ is constant, $\mathscr{F}(u)=\lambda \phi(u)+$ const. By substituting $\phi(u(x, t))$ for $U$ in (1) we have that $U(x, t)$ is a weak solution of (1) if and only if $u(x, t)$ weakly satisfies

$$
\phi(u(x, t))_{t}+\lambda \phi(u(x, t))_{x}=0 .
$$

This is a linear equation in $\phi$, and so each component of (3.53) is weakly equivalent to the linear scalar conservation law $u_{t}+\lambda u_{x}=0$, which appears in the $i$ th component. This verifies that (ii) implies (iii). Statement (i) follows from (iii) as in Theorem 1, so this completes the proof of Theorem 2.

ACKNOWLEDGEMENTS. I would like to thank James Glimm for calling my attention to the coincidence of shock and rarefaction curves in the chromatography equations, and Dan Marchesin for observing the coincidence in general concentration equations. I also thank Eli Isaacson for many helpful discussions.

\section{BIBLIOGRAPHY}

1. R. Aris and N. Amundson, Mathematical methods in chemical engineering. Vol. 2, Prentice-Hall, Englewood Cliffs, N. J.

2. R. Courant and K. O. Friedricks, Supersonic flow and shock waves, Wiley, New York, 1948.

3. J. Glimm, Solutions in the large for nonlinear hyperbolic systems of equations, Comm. Pure. Appl. Math. 18 (1965), 697-715.

4. E. Isaacson, Global solution of a Riemann problem for a non-strictly hyperbolic system of conservation laws arising in enhanced oil recovery. J. Comput. Phys. (to appear).

5. F. Helfferich and G. Klein, Multicomponent chromatography, Marcel Dekker, New York, 1970.

6. B. Keyfitz and H. Kranzer, A system of non-strictly hyperbolic conservation laws arising in elasticity theory, Arch. Rational Mech. Anal. 72 (1980).

7. P. D. Lax, Hyperbolic systems of conservation laws. II. Comm. Pure Appl. Math. 19 (1957), 537-566. 8. Shock waves and entropy, in Contributions to Nonlinear Functional Analysis (E. H. Zarantonello, editor), Academic Press, New York, 1971, pp. 603-634.

9. T. P. Liu and C. H. Wang, On a hyperbolic system of conservation laws which is not strictly hyperbolic, MRC Technical Summary Report \#2184, December 29, 1980.

10. D. W. Peaceman, Fundamentals of numerical reservoir simulation, Elsevier North-Holland, New York.

11. H. Rhee, R. Aris and N. R. Amundson, On the theon of multicomponent chromatography, Philos. Trans. Roy. Soc. London Ser A 267 (1970), 419-455. 
12. Blake Temple, Global solution of the Cauchy problem for a class of $2 \times 2$ non-strictly hyperbolic conservation laws, Adv. Appl. Math. 3 (1982), 335-375.

13. Solutions in the large for the nonlinear hyperbolic conservation laws of gas dynamics, J. Differential Equations 41 (1981), 96-161.

14. C. Conley, J. Smoller and K. Chueh, Positively invariant regions for systems of nonlinear reaction diffusion equations, Indiana Univ. Math. J. 26 (1977).

Department of Mathematical Physics, The Rockefeller University, New York, New York 10021

Current address: Math Research Center, 610 Walnut Street, Madison, Wisconsin 53705 